\title{
Meta-analysis of the relationship between the LOC387715/ARMS2 polymorphism and polypoidal choroidal vasculopathy
}

\author{
J.J. Jiang ${ }^{1,2 *}$, X. Wu ${ }^{1 *}$, P. Zhou ${ }^{1,3}$, W.Z. Yu ${ }^{1}$, L.Z. Huang ${ }^{1}$ and X.X. Li ${ }^{1}$ \\ ${ }^{1}$ Department of Ophthalmology, Peking University People's Hospital, \\ Beijing, China \\ ${ }^{2}$ Department of Ophthalmology, China-Japan Friendship Hospital, \\ Beijing, China \\ ${ }^{3}$ Department of Ophthalmology, \\ Eye and ENT Hospital of Fudan University, Shanghai, China \\ *These authors contributed equally to this study. \\ Corresponding author: X.X. Li \\ E-mail: drlixiaoxin@163.com
}

Genet. Mol. Res. 11 (4): 4256-4267 (2012)

Received June 26, 2012

Accepted October 26, 2012

Published December 17, 2012

DOI http://dx.doi.org/10.4238/2012.December.17.1

\begin{abstract}
We investigated the association between the LOC387715/ ARMS2 polymorphism (rs10490924 G>T) and susceptibility to polypoidal choroidal vasculopathy (PCV) through a meta-analysis of 1446 cases and 3255 controls from eight case-control studies. The genetic effect of the LOC387715/ARMS2 rs10490924 G>T polymorphism on PCV was assessed by calculating pooled odds ratios (ORs) with $95 \%$ confidence intervals $(95 \% \mathrm{CIs})$. We found that elevated PCV risk was significantly associated with the GG genotype (GG vs $\mathrm{TT}, \mathrm{OR}=4.23,95 \% \mathrm{CI}=3.53-5.06)$, and heterozygous genotype $\mathrm{TG}$ appeared to have a minor effect on PCV risk (TG $v s$ TT, OR $=1.47$, $95 \% \mathrm{CI}=1.26-1.71)$. Patients with the $\mathrm{T}$ allele were 2.09 times more likely to have PCV than those with the G allele $(95 \% \mathrm{CI}=1.906-2.288)$. A further subgroup analysis by ages also showed that the genetic effect of the LOC387715/ARMS2 rs $10490924 \mathrm{G}>\mathrm{T}$ polymorphism on PCV
\end{abstract}


is stronger among patients with mean age $<73$ years. Our meta-analysis strengthened the evidence that the LOC387715/ARMS2 rs10490924 $\mathrm{G}>\mathrm{T}$ polymorphism plays an important role in PCV susceptibility.

Key words: Polypoidal choroidal vasculopathy; LOC387715/ARMS2; Gene polymorphism; Meta-analysis

\section{INTRODUCTION}

Polypoidal choroidal vasculopathy (PCV) is a macular disease associated with an irreversible reduction of vision and is commonly found in the elderly population. The characteristic lesion of PCV is inner branching choroidal networks with orange polyp-like aneurismal dilation, which can be clearly demonstrated by indocyanine green angiography. Although some similarities with age-related macular degeneration (AMD), PCV has been described as a separate clinical entity differing from AMD and other macular diseases associated with subretinal neovascularization (Yannuzzi et al., 1999; Ciardella et al., 2004; Laude et al., 2010). $\mathrm{PCV}$ can occur in any gender or race, but it is more commonly seen in non-white patients and its incidence in the Asian population with AMD is as high as 20 to 55\% (Sho et al., 2003; Maruko et al., 2007).

Previous studies on the genetic basis of PCV have recognized it as a complex disease resulting from the actions and interactions of multiple genes and environmental factors. Many candidate genes have been chosen based on their prior close connection with AMD or an entire genome analysis to investigate the risk regions of PCV. Age-related maculopathy susceptibility 2 (LOC387715/ARMS2) is one of the most focused candidate genes, and various single nucleotide polymorphisms have been identified within it. Polymorphisms in LOC387715/ARMS2 were reported to decrease the stability of ARMS2 mRNA (Fritsche et al., 2008).

Empirical studies have shown positive correlations between AMD and the G>T polymorphism in the LOC387715/ARMS2 gene on chromosome 10q26 (Jakobsdottir et al., 2005; Dewan et al., 2006; Kondo et al., 2007). Recent studies have revealed that this polymorphism also contributes to the risk of PCV. However, those studies were either based on small sample size or lacked evaluation of the biological model of genetic effect. Therefore, a focused meta-analysis of current results published in the literature is greatly needed. In this paper, we combined information from eight significant publications and tried to extract powerful pieces of evidence on the correlation between the LOC387715/ARMS2 rs10490924 G>T polymorphism and susceptibility to PCV.

\section{MATERIAL AND METHODS}

\section{Search strategy}

We searched Medline, PubMed, Embase, Web of Science, and Chinese National Knowledge Infrastructure (CNKI) database using the following search terms: "age-related maculopathy susceptibility 2" or "ARMS2" or "LOC387715" or " rs10490924" or "A69s" and "genetic variant" or "polymorphism" and "polypoidal choroidal vasculopathy" or "PCV". All searched articles were manually checked to exclude any clearly irrelevant studies, and 
the remaining articles were further reviewed based on their suitability for our meta-analysis. References of the retrieved articles or preceding reviews on this topic were also screened to find additional eligible publications. For overlapping studies, only the articles with the largest sample size were included. All the search results were assessed independently by two of the authors (Jiang JJ and $\mathrm{Wu} \mathrm{X}$ ).

\section{Eligibility study and data extraction}

The eligibility criteria were: a) evaluations of the polymorphisms in the ARMS2 gene and PCV risks; b) original data of retrospective case-control or prospective cohort publications; c) the alleles and genotypes of the LOC387715/ARMS2 polymorphism (rs10490924), respectively, were G and T and GG, GT, and TT; d) sufficiency of available data for each allele or genotype in both PCV and control groups; e) fulfilling Hardy-Weinberg equilibrium (HWE) in the control group ( $P>0.01$ was eligible). Any study with inconsistent data was excluded.

The following information was extracted from selected studies: surname of the first author, publication year, country or origin, ethnicity of the studied population, characteristics of the cases and controls, number of individuals with genotypes GG, GT and TT in both cases and controls, and the methods used for genotyping. All information was carefully extracted from eligible publications independently and in duplicate by the two authors who used recommended guidelines to report on meta-analyses. Any disagreement was adjudicated by a third author.

\section{Statistical analysis}

For each study, the odds ratio (OR) with $95 \%$ confidence interval $(95 \% \mathrm{CI})$ were calculated to assess the strength of association between the LOC387715/ARMS2 rs10490924 $\mathrm{G}>\mathrm{T}$ polymorphism and PCV risk. HWE was assessed in the control group by using the $\chi^{2}$ goodness-of-fit test. Gene effect on PCV was estimated by a logistic regression approach proposed for molecular association studies. The pooled OR was calculated by the fixed effects model or the random effects model according to the heterogeneity. Heterogeneity assumption was tested by the Q-test and a $\mathrm{P}$ value greater than 0.10 indicates the lack of heterogeneity among studies. If no heterogeneity $(\mathrm{P}>0.10)$ was observed, the fixed effects model was used to calculate the pooled OR. Otherwise, the random effects model was employed. Three different types of OR were calculated: i) allele comparison ( $\mathrm{T}$ allele versus $\mathrm{G}$ allele in the LOC387715/ARMS2 rs10490924 G>T polymorphism); ii) comparison between risk-variant homozygotes and wild homozygotes (TT genotype versus GG genotype in the LOC387715/ ARMS2 rs10490924 G>T polymorphism); iii) comparison between risk-variant heterozygotes and wild homozygotes (TG genotype versus GG genotype in the LOC387715/ARMS2 rs10490924 G>T polymorphism). Population attributable risk of the risk allele was calculated according to the method described by Chang et al. (2007). The potential publication bias was assessed visually in a funnel plot of $\log (\mathrm{OR})$ against its standard error, and the degree of asymmetry was evaluated using the Egger test $(\mathrm{P}<0.05$ was considered to be statistically significant). Sensitivity analyses were carried out by including or excluding studies not in HWE, but a deviation from HWE in mixed control population was allowed (Wittke-Thompson et al., 2005). All statistical analyses were carried out with Stata/SE version 10.0 (Stata Corporation, College Station, TX, USA). 


\section{RESULTS}

\section{Study characteristics}

There were 14 published articles investigating the association between the LOC387715/ARMS2 rs10490924 G>T polymorphism and PCV risk. Two of them were kind of overlapping, and only the one with larger sample was included (Gotoh et al., 2009, 2010), four publications did not involve a case-control design (Sakurada et al., 2010, 2011; Bessho et al., 2011; Tsujikawa et al., 2011), and one was a sibling-based study (Machida et al., 2010). All remaining publications were verified for being suitable for meta-analysis. Thus, data from eight publications (Kondo et al., 2007; Lee et al., 2008; Sakurada et al., 2008; Hayashi et al., 2010; Lima et al., 2010; Nakanishi et al., 2010; Gotoh et al., 2010; Fuse et al., 2011) were included in the following analysis, to investigate the modifying effect of the rs $10490924 \mathrm{G}>\mathrm{T}$ polymorphism on PCV risk. Table 1 demonstrates the characteristics of these studies.

The sum of sample sizes of the eight studies was 4701, which included 1446 cases and 3255 controls. The average ages ranged from 63.8 to 75.1 in case groups and 46.6 to 73.2 in control groups. The gender ratio (M/F) in the two groups varied from $0.75(40 / 53)$ to $4(48 / 12)$ across the eight studies. All studies were of case-control design, with seven conducted among Asians and one in Caucasians. Cases were recruited from hospital patients and controls had mainly healthy characteristics with regard to vision and unrelated to the cases. The genotyping methods used in these studies included polymerase chain reaction, TaqMan assay, sequencing, and denaturing high-performance liquid chromatography. The HWE tests performed on controls showed that all studies fulfilled HWE (see Table 2).

\section{Allele comparison}

The frequency of the risk allele $\mathrm{T}$ in the LOC387715/ARMS2 rs10490924 G>T polymorphism in controls across all studies ranged from 0.22 to 0.39 , while the variation among Asians was smaller, ranging from 0.33 to 0.39 (Table 2). The mean $\mathrm{T}$ allele frequency in Asians was significantly higher than in Caucasians ( 0.37 versus 0.22$)$.

The association between the LOC387715/ARMS2 rs $10490924 \mathrm{G}>\mathrm{T}$ polymorphism and PCV was evaluated in the eight studies included. The heterogeneity test of the T allele versus the $\mathrm{G}$ allele $(\mathrm{OR})$ showed no statistical significance $\left(\mathrm{Q}=9.32, \mathrm{P}=0.231, \mathrm{I}^{2}=\right.$ $24.9 \%$ ); therefore, pooled OR were estimated based on the fixed effects model. As shown in Figure 1A, individuals with the $\mathrm{T}$ allele were 2.09 times more likely to have PCV than individuals with the $\mathrm{G}$ allele $(95 \% \mathrm{CI}=1.906-2.288)$. The cumulative meta-analysis results remained significant and were consistent over time (Figure 1B). No individual study affected dominantly the overall $\mathrm{OR}$ in further conducted influence analysis (OR $=2.09$, $95 \% \mathrm{CI}=1.903-2.285$ ). Moreover, a funnel plot and the Egger test were used to detect publication bias. The shape of the funnel plot did not reveal any evidence of obvious asymmetry (Figure 1C), and the Egger test also suggested an absence of publication bias in the model $(\mathrm{t}=0.84, \mathrm{P}=0.435)$. 


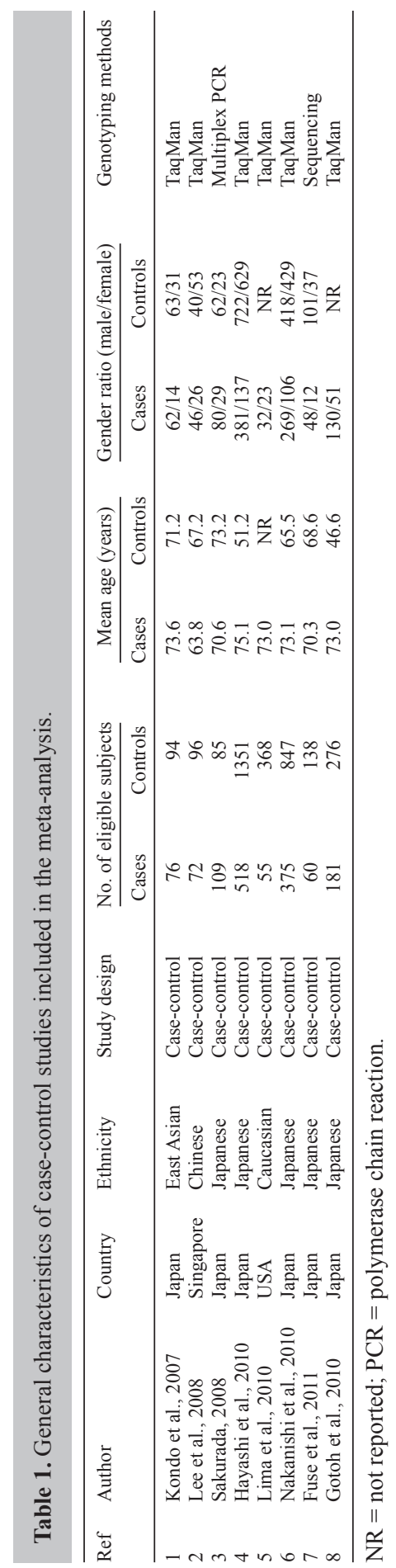


Table 2. Number of alleles and genotypes in case and control groups of studies in a meta-analysis of the association between the LOC387715 gene polymorphism and polypoidal choroidal vasculopathy.

\begin{tabular}{|c|c|c|c|c|c|c|c|c|c|c|c|}
\hline \multirow[t]{3}{*}{ Ref. } & \multirow[t]{3}{*}{ Author } & \multirow[t]{3}{*}{ Cases/controls } & \multicolumn{2}{|c|}{$\mathrm{T}$ allele frequency } & \multicolumn{6}{|c|}{ Genotype frequencies } & \multirow[t]{3}{*}{ HWE P } \\
\hline & & & \multirow[t]{2}{*}{ Cases } & \multirow[t]{2}{*}{ Controls } & \multicolumn{3}{|c|}{ Cases } & \multicolumn{3}{|c|}{ Controls } & \\
\hline & & & & & TT & TG & GG & TT & TG & GG & \\
\hline 1 & Kondo & $76 / 94$ & 0.64 & 0.38 & 33 & 31 & 12 & 15 & 41 & 38 & 0.783 \\
\hline 2 & Lee & $72 / 93$ & 0.56 & 0.39 & 25 & 30 & 17 & 12 & 48 & 33 & 0.699 \\
\hline 3 & Sakurada & $109 / 85$ & 0.64 & 0.35 & 45 & 49 & 15 & 14 & 32 & 39 & 0.269 \\
\hline 4 & Hayashi & $509 / 1336$ & 0.55 & 0.39 & 171 & 216 & 122 & 196 & 638 & 502 & 0.959 \\
\hline 5 & Lima & $55 / 368$ & 0.32 & 0.22 & 6 & 23 & 26 & 14 & 136 & 218 & 0.439 \\
\hline 6 & Nakanishi & $375 / 847$ & 0.56 & 0.37 & 133 & 152 & 90 & 113 & 401 & 333 & 0.903 \\
\hline 7 & Fuse & $60 / 138$ & 0.47 & 0.33 & 18 & 20 & 22 & 16 & 58 & 64 & 0.876 \\
\hline 8 & Gotoh & $181 / 276$ & 0.57 & 0.38 & 65 & 77 & 39 & 33 & 142 & 101 & 0.283 \\
\hline
\end{tabular}

HWE $=$ Hardy-Weinberg equilibrium.

Subgroup analysis was conducted by median (73 years) of mean ages of case groups, where a stronger effect was noted for mean age $<73$ years $(\mathrm{OR}=2.29,95 \% \mathrm{CI}$ $=1.79-2.94)$ compared to mean age $\geq 73$ years $(\mathrm{OR}=2.06,95 \% \mathrm{CI}=1.87-2.27)$ (see Table 3).

\section{Genotype comparison}

The genotype frequency of the LOC387715/ARMS2 rs10490924 G>T polymorphism between the case and control groups is presented in Table 2 . The heterogeneity test of both TT genotype versus GG genotype $\left(\mathrm{OR}_{1}\right)$ and TG genotype versus GG genotype $\left(\mathrm{OR}_{2}\right)$ showed no statistical significance $\left(\mathrm{Q}=5.90, \mathrm{P}=0.55, \mathrm{I}^{2}=0.0 \% ; \mathrm{Q}=10.04, \mathrm{P}=\right.$ $0.19, \mathrm{I}^{2}=30.3 \%$ ); therefore, pooled OR were estimated based on the fixed effects model. Overall meta-analysis indicated that the individuals who had the homozygous variant TT genotype and the heterozygous variant TG genotype had 4.23- and 1.47-fold increased risk of $\mathrm{PCV}$, respectively, compared to the individuals with the wild homozygote GG genotype $\left[\mathrm{OR}_{1}(\mathrm{TT} v s \mathrm{GG})=4.23,95 \% \mathrm{CI}=3.53-5.06 ; \mathrm{OR}_{2}(\mathrm{TG} v s \mathrm{GG})=1.47,95 \% \mathrm{CI}=\right.$ 1.26-1.71] (see Figures 2 and 3). The cumulative meta-analysis results remained significant and were consistent over time. No individual study affected the overall OR dominantly in further conducted influence analysis $\left(\mathrm{OR}_{1}=4.21,95 \% \mathrm{CI}=3.51-5.04 ; \mathrm{OR}_{2}=\right.$ $1.46,95 \% \mathrm{CI}=1.25-1.71)$. Publication bias was detected by both a funnel plot and the Egger test. The shape of the funnel plot did not reveal any evidence of obvious asymmetry, and the Egger test suggested an absence of publication bias in the model $(\mathrm{t}=1.32$, $\mathrm{P}=0.235 ; \mathrm{t}=0.97, \mathrm{P}=0.71$ ).

Moreover, we performed subgroup analysis by median ( 73 years) of the mean ages of case groups, where a stronger effect was found for both homozygous variant TT genotype and heterozygous variant TG genotype for mean age $<73$ years $\left(\mathrm{OR}_{1}=4.85,95 \% \mathrm{CI}=\right.$ 2.97-7.91; $\left.\mathrm{OR}_{2}=1.68,95 \% \mathrm{CI}=1.11-2.53\right)$ compared to mean age $\geq 73$ years $\left(\mathrm{OR}_{1}=4.13\right.$, $\left.95 \% \mathrm{CI}=3.40-5.02 ; \mathrm{OR}_{2}=1.43,95 \% \mathrm{CI}=1.21-1.69\right)($ see Table 3$)$. 
A

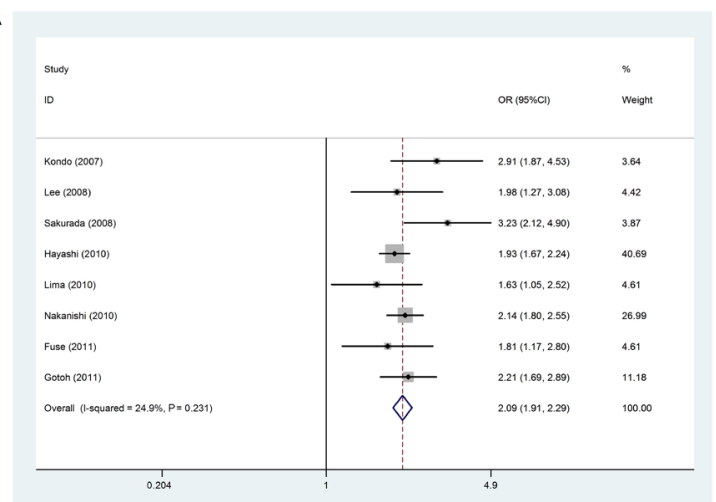

B Study

ID OR $(95 \% \mathrm{Cl})$

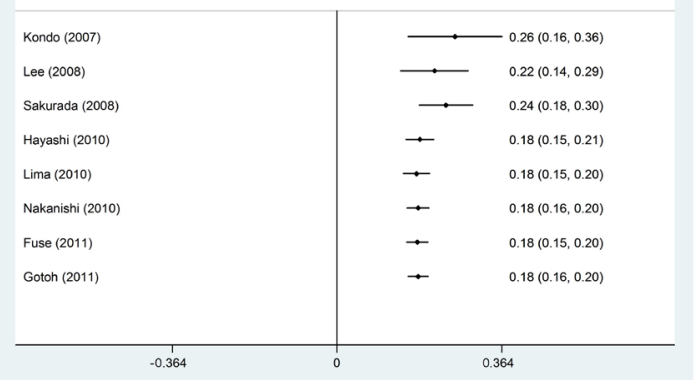

C

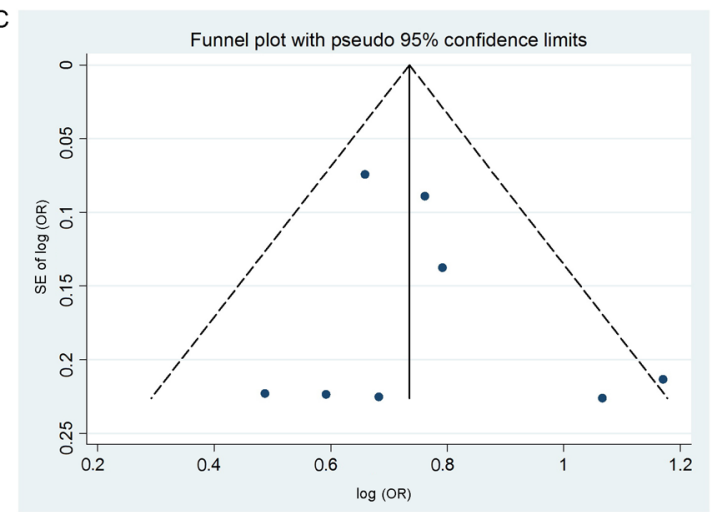

Figure 1. Forest plot, cumulative random-effects meta-analysis, and publication bias plot for meta-analysis of allele ( $\mathrm{T}$ versus $\mathrm{G}$ ) of the LOC387715/ARMS2 rs $10490924 \mathrm{G}>\mathrm{T}$ polymorphism and polypoidal choroidal vasculopathy. A. Shows the forest plot of meta-analysis. Each study is shown by the point estimate of the odds ratio (OR) (the size of the square is proportional to the weight of each study) and $95 \%$ confidence interval $(95 \% \mathrm{CI})$ for the OR (extending lines). B. Shows cumulative random-effects meta-analysis. The point indicate the OR and horizontal lines indicate $95 \% \mathrm{CI}$. C. Shows the Begg funnel plot of studies included in the meta-analysis. The horizontal axis represents $\log (\mathrm{OR})$ and the vertical axis means the standard error (SE) of log (OR). Vertical line and sloping lines in the funnel plot represent random effect summary OR and expected 95\% CI for a given SE, respectively. Each circle area represents the contribution of each study to the pooled OR. 


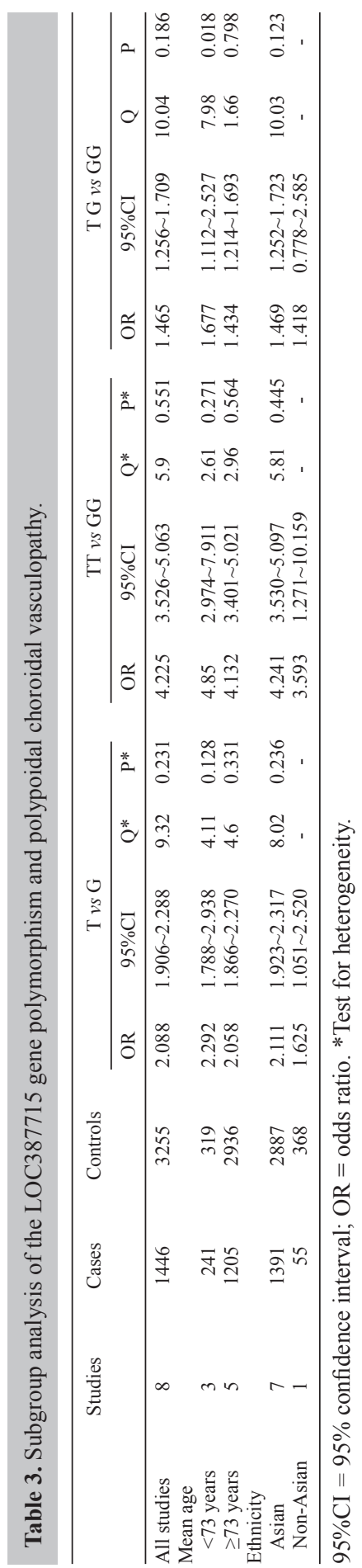




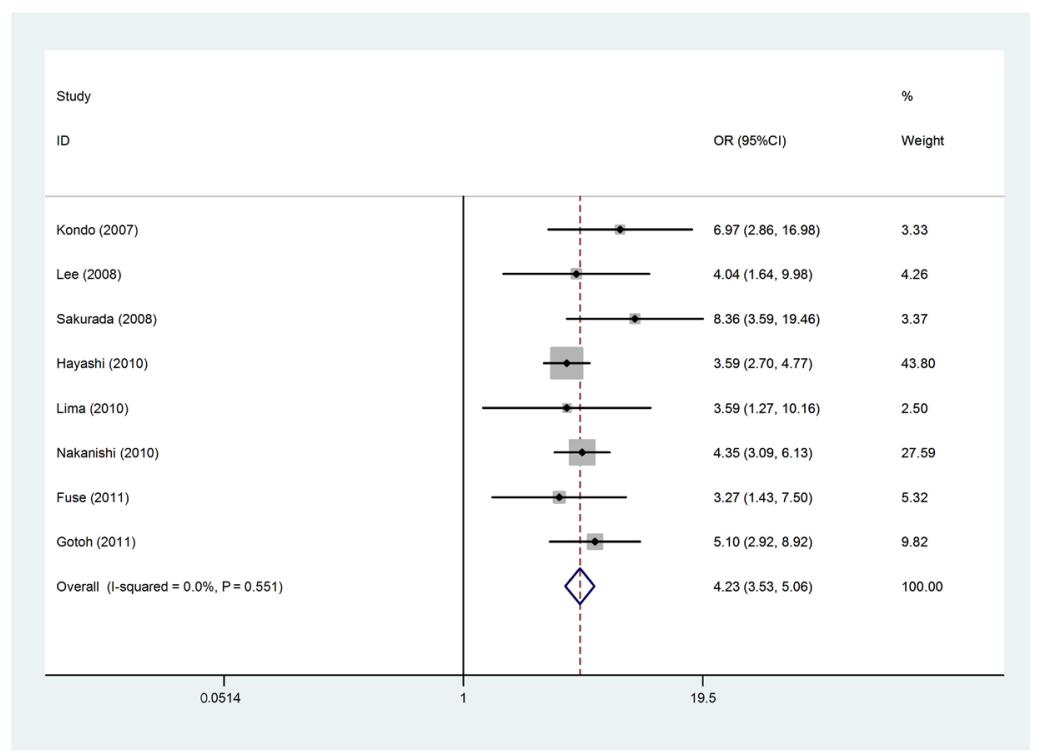

Figure 2. Random-effects meta-analysis of homozygous (TT versus GG) genotypes of the LOC387715/ARMS2 rs10490924 G>T polymorphism and polypoidal choroidal vasculopathy. Squares indicate study-specific odds ratio (OR) (size of square reflects the study-statistical weight, i.e., inverse of variance); horizontal lines indicate $95 \%$ confidence interval $(95 \% \mathrm{CI})$; diamond indicates summary OR with its corresponding 95\%CI.

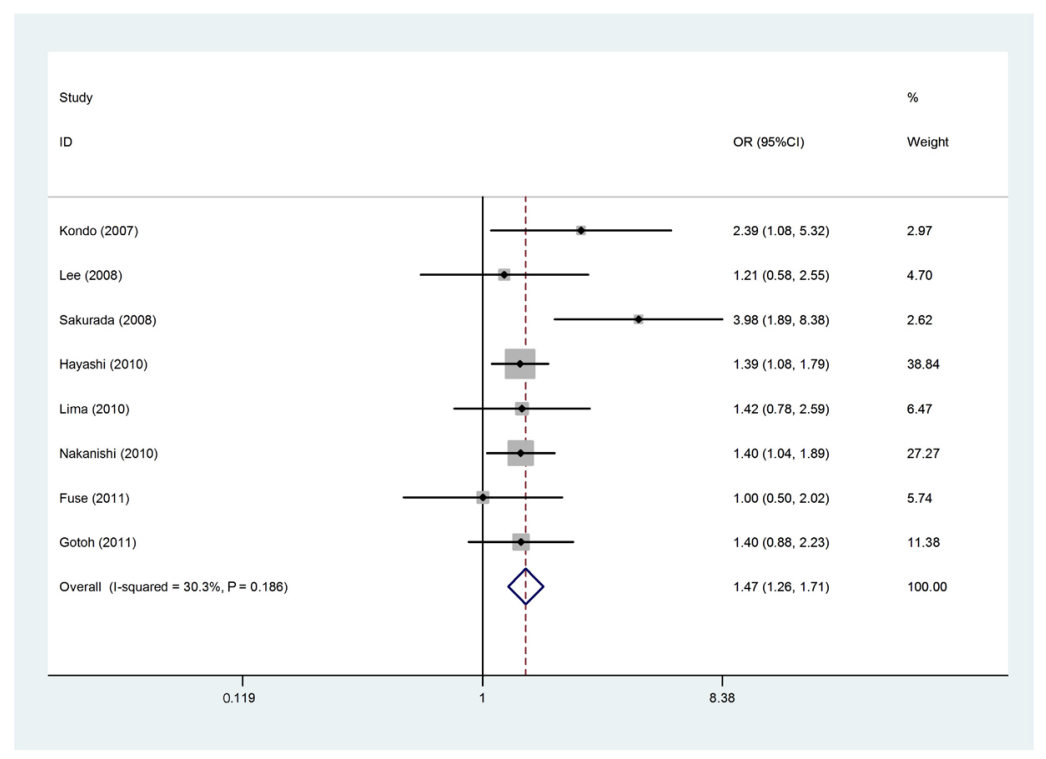

Figure 3. Random-effects meta-analysis of heterozygous (TG versus GG) genotypes of the LOC387715/ARMS2 rs10490924 G>T polymorphism and polypoidal choroidal vasculopathy. Squares indicate study-specific odds ratio (OR) (size of square reflects the study-statistical weight, i.e., inverse of variance); horizontal lines indicate $95 \%$ confidence interval $(95 \% \mathrm{CI})$; diamond indicates summary OR with its corresponding 95\%CI. 


\section{DISCUSSION}

A single nucleotide polymorphism in LOC387715/ARMS2 rs10490924 has been suggested as a possible modifier of PCV risk in a few studies. The present study for the first time looks into this issue using meta-analysis. Our study showed a strong genetic effect of the LOC387715/ARMS2 rs10490924 G>T polymorphism on PCV and indicated a moderate codominant mode of action.

It is widely accepted that the LOC387715/ARMS2 gene polymorphisms are significantly associated with the phenotype and progression of AMD (Rivera et al., 2005; Dewan et al., 2006; Yoshida et al., 2007). PCV is usually clinically categorized as a subtype of neovascular AMD because it has been determined that PCV and AMD have considerable overlaps in molecular gene determinants, genetic background, pathophysiological characteristics, clinical manifestations, and environmental risk factors (Ciardella et al., 2004; Maruko et al., 2007; Laude et al., 2010). Our primary analysis indicated that, for the LOC387715/ARMS2 rs10490924 G>T polymorphism, homozygosity and heterozygosity for the at-risk allele genotype (TT and TG) increased the likelihood for PCV 4.2- and 1.5-fold, respectively, compared to the wild-type genotype. This finding confirmed the remarkable genetic effect of the LOC387715/ARMS2 gene polymorphism and provided additional evidence of the common genetic background shared between PCV and AMD.

The LOC387715/ARMS2 gene has been shown to be expressed in the mitochondria of retina photoreceptors and may be associated with RPE dysfunction (Fritsche et al., 2008). The lesion of RPE may subsequently lead to abnormal choroidal vasculopathy, which results in PCV. In addition, chromosome 10q26 has been reported to harbor three important AMD susceptible genes, including Pleckstrin homology domain-containing protein family A member 1, age-related maculopathy susceptibility 2 (LOC387715/ARMS2), and high-temperature requirement factor A 1 (HTRA1/PRSS11) (Jakobsdottir et al., 2005; Rivera et al., 2005). Previous studies have shown the distinct association between the HTRA1 polymorphism and PCV (Kondo et al., 2007; Lee et al., 2008; Gotoh et al., 2010) and proved the strong linkage disequilibrium (LD) across the ARMS2/HTRA1 region (Laude et al., 2010). Therefore, the T allele is possibly in LD with the causative allele, which may further contribute to the significant effect of the LOC387715/ARMS2 rs10490924 G>T polymorphism on PCV.

The present meta-analysis shows that the genetic effect of the LOC387715/ARMS2 rs10490924 G>T polymorphism on PCV was more significant among patients of mean age $<73$ years than among patients of mean age $\geq 73$ years. This conclusion is inconsistent with previous studies, which have shown that the older age subgroup has a stronger effect of the HTRA1 polymorphism on AMD (Leske et al., 2006; Tang et al., 2009). Although age is an acceptable environmental risk factor for PCV (Yannuzzi et al., 1999; Ciardella et al., 2004), a few studies indicated that PCV was most commonly diagnosed among the patients with ages between 60 and 70 years (Ciardella et al., 2004; Imamura et al., 2010). It is also possible that PCV does not have a steep slope for age-specific increase in incidence, which is different from AMD. Our analysis confirms that environmental factors can also play an important role in PCV development. Seven of eight publications were based on Asian populations, which may be explained by the asymmetry incidence of PCV in different ethnicities. According to previous research, PCV tends to occur more frequently in dark-pigmented individuals, such as black patients and Asians (Sho et al., 2003), and the incidence of PCV seems to be remark- 
ably high in Japanese (Maruko et al., 2007). Thus, our meta-analysis results reinforced the conclusion that the effect of the LOC387715/ARMS2 rs10490924 G>T polymorphism on susceptibility to PCV is particularly important for Asians.

Some limitations of our meta-analysis should be acknowledged. First, the numbers of non-Asian patients included from the selected studies in our meta-analysis were relatively small to perform reliable subgroup analysis by ethnicity. Second, the unavailability of more detailed information of target patients prevented a more elaborative evaluation over adjusted OR by age, gender, environmental factors, and other potentially suspected factors (DeAngelis et al., 2007). Third, further explorations of gene-gene or gene-environment interactions and gene-subtype PCV associations were limited due to the lack of original data.

In summary, our meta-analysis provided evidence that the LOC387715/ARMS2 rs $10490924 \mathrm{G}>\mathrm{T}$ polymorphism is strongly associated with a PCV risk. The genetic effect on PCV was more significant among individuals of mean age $<73$ years and particularly played a more important role among Asians. Further well-designed studies with larger sample size, less ethnic bias and better control of suspected factors are necessary to fully evaluate the genetic contributions and confirm these findings.

\section{ACKNOWLEDGMENTS}

Research supported by the National Basic Research Program of China (973 Program; \#2011CB510200).

\section{REFERENCES}

Bessho H, Honda S, Kondo N and Negi A (2011). The association of age-related maculopathy susceptibility 2 polymorphisms with phenotype in typical neovascular age-related macular degeneration and polypoidal choroidal vasculopathy. Mol. Vis. 17: 977-982.

Chang YC, Chang TJ, Jiang YD, Kuo SS, et al. (2007). Association study of the genetic polymorphisms of the transcription factor 7-like 2 (TCF7L2) gene and type 2 diabetes in the Chinese population. Diabetes 56: 2631-2637.

Ciardella AP, Donsoff IM, Huang SJ, Costa DL, et al. (2004). Polypoidal choroidal vasculopathy. Surv. Ophthalmol. 49: 25-37.

DeAngelis MM, Ji F, Kim IK, Adams S, et al. (2007). Cigarette smoking, CFH, APOE, ELOVL4, and risk of neovascular age-related macular degeneration. Arch. Ophthalmol. 125: 49-54.

Dewan A, Liu M, Hartman S, Zhang SS, et al. (2006). HTRA1 promoter polymorphism in wet age-related macular degeneration. Science 314: 989-992.

Fritsche LG, Loenhardt T, Janssen A, Fisher SA, et al. (2008). Age-related macular degeneration is associated with an unstable ARMS2 (LOC387715) mRNA. Nat. Genet. 40: 892-896.

Fuse N, Mengkegale M, Miyazawa A, Abe T, et al. (2011). Polymorphisms in ARMS2 (LOC387715) and LOXL1 genes in the Japanese with age-related macular degeneration. Am. J. Ophthalmol. 151: 550-556.

Gotoh N, Nakanishi H, Hayashi H, Yamada R, et al. (2009). ARMS2 (LOC387715) variants in Japanese patients with exudative age-related macular degeneration and polypoidal choroidal vasculopathy. Am. J. Ophthalmol. 147: 103741, 1041.

Gotoh N, Yamashiro K, Nakanishi H, Saito M, et al. (2010). Haplotype analysis of the ARMS2/HTRA1 region in Japanese patients with typical neovascular age-related macular degeneration or polypoidal choroidal vasculopathy. Jpn. J. Ophthalmol. 54: 609-614.

Hayashi H, Yamashiro K, Gotoh N, Nakanishi H, et al. (2010). CFH and ARMS2 variations in age-related macular degeneration, polypoidal choroidal vasculopathy, and retinal angiomatous proliferation. Invest. Ophthalmol. Vis. Sci. 51: 5914-5919.

Imamura Y, Engelbert M, Iida T, Freund KB, et al. (2010). Polypoidal choroidal vasculopathy: a review. Surv. Ophthalmol. 55: 501-515. 
Jakobsdottir J, Conley YP, Weeks DE, Mah TS, et al. (2005). Susceptibility genes for age-related maculopathy on chromosome 10q26. Am. J. Hum. Genet. 77: 389-407.

Kondo N, Honda S, Ishibashi K, Tsukahara Y, et al. (2007). LOC387715/HTRA1 variants in polypoidal choroidal vasculopathy and age-related macular degeneration in a Japanese population. Am. J. Ophthalmol. 144: 608-612.

Laude A, Cackett PD, Vithana EN, Yeo IY, et al. (2010). Polypoidal choroidal vasculopathy and neovascular age-related macular degeneration: same or different disease? Prog. Retin. Eye Res. 29: 19-29.

Lee KY, Vithana EN, Mathur R, Yong VH, et al. (2008). Association analysis of CFH, C2, BF, and HTRA1 gene polymorphisms in Chinese patients with polypoidal choroidal vasculopathy. Invest. Ophthalmol. Vis. Sci. 49: 26132619.

Leske MC, Wu SY, Hennis A, Nemesure B, et al. (2006). Nine-year incidence of age-related macular degeneration in the Barbados Eye Studies. Ophthalmology 113: 29-35.

Lima LH, Schubert C, Ferrara DC, Merriam JE, et al. (2010). Three major loci involved in age-related macular degeneration are also associated with polypoidal choroidal vasculopathy. Ophthalmology 117: 1567-1570.

Machida S, Takahashi T, Gotoh N, Yoshimura N, et al. (2010). Monozygotic twins with polypoidal choroidal vasuculopathy. Clin. Ophthalmol. 4: 793-800.

Maruko I, Iida T, Saito M, Nagayama D, et al. (2007). Clinical characteristics of exudative age-related macular degeneration in Japanese patients. Am. J. Ophthalmol. 144: 15-22.

Nakanishi H, Yamashiro K, Yamada R, Gotoh N, et al. (2010). Joint effect of cigarette smoking and CFH and LOC387715/ HTRA1 polymorphisms on polypoidal choroidal vasculopathy. Invest. Ophthalmol. Vis. Sci. 51: 6183-6187.

Rivera A, Fisher SA, Fritsche LG, Keilhauer CN, et al. (2005). Hypothetical LOC387715 is a second major susceptibility gene for age-related macular degeneration, contributing independently of complement factor $\mathrm{H}$ to disease risk. Hum. Mol. Genet. 14: 3227-3236.

Sakurada Y, Kubota T, Mabuchi F, Imasawa M, et al. (2008). Association of LOC387715 A69S with vitreous hemorrhage in polypoidal choroidal vasculopathy. Am. J. Ophthalmol. 145: 1058-1062.

Sakurada Y, Kubota T, Imasawa M, Mabuchi F, et al. (2010). Association of LOC387715 A69S genotype with visual prognosis after photodynamic therapy for polypoidal choroidal vasculopathy. Retina 30: 1616-1621.

Sakurada Y, Kubota T, Imasawa M, Mabuchi F, et al. (2011). Role of complement factor H I62V and age-related maculopathy susceptibility 2 A69S variants in the clinical expression of polypoidal choroidal vasculopathy. Ophthalmology 118: 1402-1407.

Sho K, Takahashi K, Yamada H, Wada M, et al. (2003). Polypoidal choroidal vasculopathy: incidence, demographic features, and clinical characteristics. Arch. Ophthalmol. 121: 1392-1396.

Tang NP, Zhou B, Wang B and Yu RB (2009). HTRA1 promoter polymorphism and risk of age-related macular degeneration: a meta-analysis. Ann. Epidemiol. 19: 740-745.

Tsujikawa A, Ojima Y, Yamashiro K, Nakata I, et al. (2011). Association of lesion size and visual prognosis to polypoidal choroidal vasculopathy. Am. J. Ophthalmol. 151: 961-972.

Wittke-Thompson JK, Pluzhnikov A and Cox NJ (2005). Rational inferences about departures from Hardy-Weinberg equilibrium. Am. J. Hum. Genet. 76: 967-986.

Yannuzzi LA, Wong DW, Sforzolini BS, Goldbaum M, et al. (1999). Polypoidal choroidal vasculopathy and neovascularized age-related macular degeneration. Arch. Ophthalmol. 117: 1503-1510.

Yoshida T, Dewan A, Zhang H, Sakamoto R, et al. (2007). HTRA1 promoter polymorphism predisposes Japanese to agerelated macular degeneration. Mol. Vis. 13: 545-548. 